\title{
SOME PROPERTIES OF PARTLY-ASSOCIATIVE OPERATIONS
}

\section{H. A. THURSTON}

\section{Introduction.}

Summary. Three properties of a group-operation are

(i) it is associative: $(x y) z=x(y z)$;

(ii) it is regular: $a=b$ if $a x=b x$ or if $y a=y b$; and

(iii) it is reversible: $a x=y a=b$ is solvable for $x$ and $y$.

These definitions may readily be generalized. For example, the associative property may be stated as "the two continued products which can be formed from the same three elements in the same order are equal (for all values of the elements concerned)." Under a $(\nu+1)$-ary operation, $\nu+1$ continued products can be formed from $2 \nu+1$ elements in order. For any given operation, some, none, or all of these may be equal. If some are equal, the operation is partlyassociative. If in addition the operation is regular and reversible, then there are numbers $j$ and $k, \nu$ being a multiple of $k$ and $k$ of $j$, such that the $p$ th continued product is equal to the $(p+q)$ th if $p$ is a multiple of $j$ and $q$ of $k$. (Partly associative operations, J. London Math. Soc. vol. 24 (1949) pp. 260-271.) Such an operation is $(j, k)$-associative. If $j=k=1$ (that is, if all the continued products are equal) the operation is, if reversible, that of a polyadic group. (E. L. Post, Polyadic groups, Trans. Amer. Math. Soc. vol. 48 (1940) pp. 208350.)

A fundamental theorem about polyadic groups is that a polyadic operation can be regarded as the continued product of a group operation. (Op. cit. pp. 218-219.) The proof of this involves setting up an equivalence such that an ordered set can replace any equivalent ordered set in a polyadic product without changing the value of the product. (Op. cit. p. 217.) The continued-product theorem can be generalized to apply to $(1, k)$-associative operations (Theorem $\mathrm{H}$ of the present paper) and the replacement theorem to $(j, k)$-associative operations (Theorem E). Other replacement theorems are proved in part 2. They do not require full reversibility and I have stated them with only the properties actually required for the proofs. They can be summed up (in somewhat less general forms than in the text) as follows:

Let $(\alpha, \beta, \gamma)$ be either $(1,-1,1),(0,0,1),(0,1,0)$, or $(1,0,0)$. Then if $*$ is a 0 - and $\nu$-reversible $(j, k)$-associative operation, if

Received by the editors November 13, 1952. 
$p \equiv p^{\prime} \equiv \alpha, q \equiv \beta$, and $r \equiv r^{\prime} \equiv \gamma$ modulo $j$, if $p+q+r=p^{\prime}+q+r^{\prime}=\nu+1$, and if either $p$ or $r$ is congruent to $\alpha$ modulo $k$, then

$$
* a_{1} \cdots a_{p} f_{1} \cdots f_{q} c_{1} \cdots c_{r}=* a_{1} \cdots a_{p} g_{1} \cdots g_{q} c_{1} \cdots c_{r}
$$

implies that

$$
* b_{1} \cdots b_{p^{\prime}} f_{1} \cdots f_{q} d_{1} \cdots d_{r^{\prime}}=* b_{1} \cdots b_{p^{\prime}} g_{1} \cdots g_{q} d_{1} \cdots d_{r^{\prime}} .
$$

This is also true if $q \equiv 1(\bmod k)$ and $p=0 \equiv p^{\prime}$ or $r=0 \equiv r^{\prime}(\bmod j)$.

Most of the theorems hold not only for elements $f_{i}, g_{i}$, but also for sets of elements, using an analogue of Kronecker multiplication.

The case $\nu=1$ of Theorem B is the well-known result that if, in a group, $e a=a$ for some $a$, then $x e=x$ for all $x$. A set $e_{1} \cdots e_{\nu}$ of elements such that $* e_{1} \cdots e_{\nu} x=x$ for all $x$ may accordingly be called a polyadic left-identity. Part 3 of the paper consists of theorems similar to Theorem $\mathrm{B}$, including the results that if $* e_{1} \cdots e_{\nu} a=a$ for some $a$, then $e_{1} \cdots e_{\nu}$ is a left-identity; that if $e_{1} \cdots e_{\nu}$ is a left-identity and, $m$ being a multiple of $k$, the first $m$ elements are taken from the front to the back (giving $e_{m+1} \cdots e_{\nu} e_{1} \cdots e_{m}$ ), we still have a leftidentity; and that $e_{1} \cdots e_{\nu}$ is a left-identity if and only if $e_{2} \cdots e_{\nu} e_{1}$ is a right-identity (that is, $* x e_{2} \cdots e_{\nu} e_{1}=x$ for all $x$ ).

The "factorizing" of the partly-associative operation into the continued product of a shorter partly-associative operation may be contrasted with the factorizing of a general operation in Theorem 12 of The structure of an operation, J. London Math. Soc. vol. 27 (1952) pp. 271-279.

Definitions and Notation. A $(\nu+1)$-ary operation is a mapping of a power $S^{\nu+1}$ of a set $S$ into $S$. In this paper $\nu$ is always finite.

Elements of $S$ are denoted by $z, y, x, \cdots$ in statements which are true for all selections of these elements from $S$; and by $a, b, c, \cdots$ where this may not be so.

Capital letters denote sets of elements.

$i, j, k, l, m, n, p, q, r$, and $\nu$ denote integers.

Signs such as $\dagger$ or $*$ denote operations.

German letters denote equivalences: $x q$ is the set of all elements $y$ for which $x q y$.

Frequent use will be made of ordered sets. If $q \geqq p$, then $x_{q}^{p}$ denotes $x_{p} x_{p+1} \cdots x_{q}$; and $x_{p-1}^{p}$ denotes the null set. The formula $x_{q}^{p}$ is invalid if $q<p-1$. An element, together with its suffixes, affixes, and so on, is treated as one entity. For example, $a_{1, q}^{p}$ denotes $a_{1, p} \cdots a_{1, q}$, and $\left(x_{q}^{p} q\right)$ denotes $\left(x_{p} q \cdots x_{q} q\right)$. When the range of the suffix is obvious, $x_{a}^{p}$ is abbreviated to $\mathbf{x}$. 
The product of $x_{\nu}^{0}$ under $*$ is $* x_{\nu}^{0}$ or $*_{x}$. The continued product of $x_{2 \nu}^{0}$ (i.e. the product of $* x_{\nu}^{0}$ and $x_{2 \nu}^{\nu+1}$ ) is $*^{2} x$, and so on.

If $Y$ are elements or sets of elements of $S$, then ${ }^{*} Y$ is the set of all $*_{y}$ for which $y_{i}=Y_{i}$ if $Y_{i}$ is an element, and $y_{i} \in Y_{i}$ if $Y_{i}$ is a set of elements.

If, given any $x_{l-1}^{0}, y$, and $z$ of $S$, there is an $a$ of $S$ for which $* x_{l-1}^{0} a y$ $=z$; then $*$ is $l$-reversible. We shall consider operations which are $l$-reversible for some value of $l$; it will not matter which, except that the extreme case $(l=0$ or $l=\nu)$ is not enough on its own. This suggests the definition: $*$ is once-reversible if it is either $l$-reversible for some $l$ for which $0<1<\nu$ or both 0 -reversible and $\nu$-reversible.

If $b=d$ whenever $* a_{l-1}^{0} b c=* a d c$, then $*$ is $l$-regular.

If $\nu$ is a multiple of $k$ and $k$ of $j$, if $*$ is $(\nu+1)$-ary, and if $* x_{p-1}^{0} * x_{2 \nu}^{p}$ $=x_{q-1}^{0} * x_{2 \nu}^{q}$ whenever $p$ is a multiple of $j$ and $q-p$ of $k$, then $*$ is $(j, k)$ associative.

\section{A generalization of a theorem of E. L. Post.}

A. THEOREM. If $a(\nu, \nu)$-associative $(\nu+1)$-ary operation is oncereversible then it is 0-regular and $\nu$-regular.

Proof. If $* f a=* g a$, let $*^{2} f a b=f$ and $* c f=g$. (If $*$ is $l$-reversible where $0<l<\nu$, we can suitably choose $b_{l}$ and $c_{l}$. If not, we can choose $b_{\nu}$ and $c_{0}$.)

Then $f=*^{2} f a b=*^{2} g a b=*^{3} c f a b=* c^{2} f a b=* c f=g$. Therefore $*$ is 0 -regular. Similarly, it is $\nu$-regular.

Note. The case $\nu=1$ is the theorem that in a group $a x=b$ and $x a=b$ are uniquely solvable.

B. Theorem. If a $(\nu+1)$-ary $(j, k)$-associative operation $*$ is either 0 -regular or $(\nu-m)$-regular, and if $m$ is a nonzero multiple of $k$, then $* e_{\nu}^{1} a=a$ implies that $* x e_{\nu}^{\nu-m+2} e_{\nu-m+1}^{1}=x$.

Proof. For any $f_{\nu-m}^{1}$,

$$
\begin{aligned}
* x e_{\nu}^{\nu-m+2} a f & =* x e_{\nu}^{\nu-m+2} * e_{\nu}^{1} a f \\
& =*^{2} x e_{\nu}{ }^{\nu+m+2} e_{\nu}{ }_{\nu} a f
\end{aligned}
$$

(because $*$ is $(j, k)$-associative).

Therefore, if $*$ is 0 -regular,

$$
x=* x e_{\nu}^{\nu-m+2} e_{\nu-m+1}^{1} .
$$

And, for any $f_{\nu-m}^{1}$,

$$
\begin{aligned}
* f x e_{\nu}^{\nu-m+2} a & =* f x e_{\nu}^{\nu-m+2}{ }^{\nu 1} e_{\nu} a \\
& =* f * x e_{\nu}^{\nu-m+2} e_{\nu}{ }_{\nu} a \quad \text { (because } * \text { is }(j, k) \text {-associative). }
\end{aligned}
$$


Therefore, if $*$ is $(\nu-m)$-regular, $x=* x e_{\nu}^{\nu-m+2} e_{\nu-m+1}^{1}$.

Note. Similarly, if $*$ is $(\nu+1)$-ary, $(j, k)$-associative, and either $\nu$ or $m$-regular, and if $m$ is a nonzero multiple of $k$, then $* a e_{\nu}^{1}=a$ implies that $* e_{\nu}^{m} e_{m-1}^{1} x=x$.

C. Lemma. If $*$ is $(\nu+1)$-ary, 0 -regular, $l$-reversible with $l<\nu$, and $(j, k)$-associative, if $p$ is a nonzero multiple of $k$, and if $d_{\nu-p}^{1}$ are any elements, then, for some $e_{p}^{1}, * x d e=x$.

Proof. Case (i). $l=0 . *$ is now 0 -reversible and so, for some $e_{k}$, $* e_{p}^{k} d e_{\mathbf{k}-1}^{1} a=a$. Therefore $* x \boldsymbol{d} e_{p}^{1}=x$, by Theorem $\mathrm{B}$.

Case (ii). $\nu-k+1 \leqq l \leqq \nu-1$. Clearly $p \geqq k$, and so $\nu-p+1 \leqq l \leqq \nu-1$. Therefore the element in position $l$ in the product $* e_{p} d e_{p-1}^{1} a$ is one of the $e^{\prime}$ s-in fact, $e_{l-p+p}$. For some value of this element the product is equal to $a$, because $*$ is $l$-reversible. Then, by Theorem $\mathrm{B}$ (with $m=\nu), * x \boldsymbol{d} e_{p}^{1}=x$.

Case (iii).

$$
1 \leqq l \leqq \nu-k .
$$

Let $r$ be the least non-negative integer for which $l \leqq p+r k$. Put $m=\nu-p-r k$. By (1), $l \leqq \nu-k$ and so, $\nu-k$ being a multiple of $k$, the least multiple of $k$ not less than $l$ is not greater than $\nu-k$. That is, $p+r k \leqq \nu-k$. Therefore $k \leqq \nu-p-r k=m$, and so $m \geqq 1$. Clearly $m=\nu-p-r k \leqq \nu-p+1$, and so $\nu-p \geqq m-1$. Therefore the formula $* d_{\nu-p}^{m} e_{p}^{1} d_{m-1}^{1} a$ is valid. If $r>0$, we have, by the definition of $r, p+(r-1) k+1 \leqq l$. Therefore $l-1 \geqq p+(r-1) k \geqq r k$. And if $r=0$ we see from (1) that $l-1 \geqq r k$. Therefore whatever $r$ is, $\nu-p-(l-1)$ $\leqq \nu-p-r k=m$. Therefore

$$
\nu-p-m+1 \leqq l .
$$

Now, by the definition of $r, l \leqq p+r k$. Therefore $\nu-l \geqq \nu-p-r k=m$, and so

$$
l \leqq \nu-m .
$$

From (2) and (3), the element in position $l$ in $* d_{\nu-p}^{m} e_{p}^{1} d_{m-1}^{1} a$ is one of the $e$ 's. Therefore for some $e, * d_{\nu-p}^{m} e_{p}^{1} d_{m-1}^{1} a=a$. Therefore, by Theorem $\mathrm{B}, * x \mathrm{de}=x$.

D. Lemma. If * is $(\nu+1)$-ary, $(j, k)$-associative, l-reversible with $l<\nu$, and 0 -regular, and if $p$ is a nonzero multiple of $k$, and $q$ a multiple of $j$, and if $* F_{p}^{0} a_{\nu-p}^{1}=* G_{p}^{0} a$, then $* b_{q}^{1} F d=* b_{q}^{1} G d$.

Proof. By Lemma $\mathrm{C}$, there is an $e$ such that $* x a e=x$. Then 


$$
\begin{aligned}
* b F d & =* b F_{p-1}^{0} * F_{p} \text { aed } \\
& =* b * F a e d \\
& =* b * G a e d \\
& =* b G_{p-1}^{0} * \text { Gaed } \\
& =* b G d
\end{aligned}
$$

(because $*$ is $(j, k)$-associative)

$$
\text { (because } * F a=* G a \text { ) }
$$

(because $*$ is $(j, k)$-associative) (because $* x$ ae $=x$ ).

Note. The set of all $(F, G)$ such that there is an a for which $* F a=* G a$ is now clearly an equivalence.

E. Theorem. If $*$ is $(\nu+1)$-ary, $(j, k)$-associative, l-reversible with $l<\nu$, and 0-regular, and if $q$ is a multiple of $j$ and $p$ of $k$, and if $* F_{p}^{0} a_{\nu-p}^{1}$ $=* G_{p}^{0} a$, then $* b_{q}^{1} F d=* b G d$.

Proof. If $p \neq 0$, this is Lemma D. If $p=0$ we have $* F_{0} a=* G_{0} a$, and $*$ is 0 -regular. Therefore $F_{0}=G_{0}$. Therefore $* b F_{0} d=* b G_{0} d$.

F. Putting $j=1$ in Theorem E:

COROLlARY. If * is $(\nu+1)$-ary, $(1, k)$-associative, and once-reversible, and if $p$ is a multiple of $k$, and if $* f_{p}^{0} a_{\nu-p}^{1}=* g_{p}^{0} a$, then $* b f d=* b g d$ for any $b$ and $d$.

Proof. By Theorem A, * is 0-regular, and the conditions of Theorem $E$ are then satisfied.

G. Lemma. If * is $(\nu+1)$-ary, $(1, k)$-associative, and once-reversible, if $\mathfrak{q}$ is the set of all $\left(f_{p}^{0}, g_{p}^{0}\right)$ for which $p$ is a multiple of $k$ and for which there is an a such that $* f a=* g a$, and if, for $i$ from 0 to $k, f_{i, p_{i}} \mathfrak{q} g_{i, p_{i}}$, then ${ }^{*} f_{0, p_{0}}^{0} \cdots f_{k, p_{k}}^{0} q^{* r} g_{0, p_{0}}^{0} \cdots g_{k, p_{k}}^{0}$ where $r$ is the greatest integer for which

$$
k+\sum_{0}^{k} p_{i} \geqq r \nu+1 \text {. }
$$

(The reason for taking this value of $r$ is that there are $k+\sum_{0}^{\boldsymbol{k}} p_{i}$ elements in $f_{0, p_{0}}^{0} \cdots f_{k, p_{k}}^{0}$, and $r \nu+1$ elements in an $r$-fold continued product.)

PROOF. For any $a_{q}^{1}$, where $q=(r+1) \nu+1-k-\sum_{0}^{k} p_{i}$, we have

$$
\begin{aligned}
*^{r+1} f_{0, p_{0}}^{0} & \cdots f_{k, p_{k} a}^{0} \\
& =*^{r+1} g_{0, p_{0}}^{0} f_{1, p_{1}}^{0} \cdots a \\
& =*^{r} g_{0, p_{0}-1}^{0} * g_{0, p_{0}} f_{1, p_{1}}^{0} \cdots a \\
& =*^{r} g_{0, p_{0}-1}^{0} * g_{0, p_{0}} g_{1, p_{1}}^{0} \cdots a
\end{aligned}
$$

and so on until 


$$
\begin{aligned}
& =*^{r}{ }_{0, p_{0}}^{0} \cdots * \cdots \stackrel{g}{g}, p^{0} \mathbf{a} \\
& =*^{r+1}{ }_{g_{0, p_{0}}}^{0} \cdots g_{k, p_{k}}^{0} \mathbf{a} .
\end{aligned}
$$

H. MaIN Theorem. If $*$ is an $(n k+1)$-ary, $(1, k)$-associative, oncereversible operation on $S$, then there is a $(k, k)$-associative $(k+1)$-ary operation $\dagger$ on a set $U$ containing $S$ such that $\dagger^{n} \mathbf{x}=* \mathbf{x}$ for every $\mathbf{x}$ of $S^{n k+1}$.

Proof. Let $q$ be as in Lemma $G$, and let $T$ be

$$
\bigcup_{n>m \geqq 0} S^{m k+1} / \mathfrak{q} \text {. }
$$

We define an operation $\dagger$ on $T$ as follows. Let $t_{\mathbf{k}}^{0}$ be any $k+1$ elements of $T$. $t_{0}$ will be of the form $\left(x_{m_{1}}^{0}\right) q$, where $m_{1}$ is a multiple of $k$. Then $t_{1}$ is of the form $\left(x_{m_{2}}^{m_{1}+1}\right) q$, where $m_{2}-1$ is a multiple of $k$. And in general $t_{i}$ is of the form $\left(x_{m_{i+1}}^{m_{i}+1}\right) \mathfrak{q}$, where $m_{i}-(i-1)=j_{i} k$. Now put $\dagger t_{\mathbf{k}}^{0}=\left({ }^{r} x_{m_{k+1}}^{0}\right) \mathfrak{q}$, where $r$ is the greatest integer for which $r \nu \leqq m_{k+1}$. Then $\dagger$ is a relation on $T^{k+1}$ into $T$. To be an operation on $T$, it must be a mapping. That is: if, for every $i,\left(x_{m_{i+1}}^{m_{i}+1}\right) \mathfrak{q}=\left(y_{m_{i+1}}^{m_{i}+1}\right) \mathfrak{q}$, then $\left({ }^{*} x_{m_{k+1}}^{0}\right) \mathfrak{q}=\left({ }^{*} y_{m_{k+1}}^{0}\right) \mathfrak{q}$; for, if not, $t t_{\boldsymbol{k}}^{0}$ would not be uniquely determined. Lemma $\mathrm{G}$, however, ensures that this is so. Therefore $\dagger$ is a $(k+1)$-ary operation on $T$.

If every $j_{i}=0$, then $t_{i}=x_{i} q$ and so, from the definition of $\dagger, \dagger x_{\mathbf{k}}^{0} q$ $=\left(x_{k}^{0}\right) \mathfrak{q}$. Then

$$
\dagger^{n} x_{n k}^{0} \mathfrak{q}=\left(* x_{n k}^{0}\right) \mathfrak{q}
$$

Now

$$
\begin{aligned}
& \dagger t_{k-1}^{0} \dagger t_{2 k}^{k}=\left(*^{p} x_{m_{k}}^{0} *^{q} x_{m_{2 k+1}}^{m_{k+1}}\right) q \\
& =\left(*^{p+q} x_{m_{2 k+1}}^{0}\right) \mathfrak{q} \quad \text { (because } m_{k}+1=j_{k} k+k-1+1 \text { and so is } \\
& \text { a multiple of } k \text { ) } \\
& =t+t_{2 k}^{0}
\end{aligned}
$$

That is, $\dagger$ is $(k, k)$-associative.

All we have to do now is to replace $T$ by a set which contains $S$, and $\dagger$ will have all the required properties. (This is easy because, although $T$ does not contain $S$, it contains, as we shall see, the set of all $\{x\}$ for all elements $x$ of $S$, where $\{x\}$ denotes the set whose only element is $x$.)

By Theorem A, * is 0-regular. Therefore $x=y$ if and only if $x q=y q$. Therefore $x q=\{x\}$. Let $U$ be $S \cup \cup_{n>m>0} S^{m k+1} / q$ : that is, $T$ with $x$ 
in place of $x \mathfrak{q}$ everywhere. Because $x q=\{x\}$, we can define $\dagger$ as a $(k+1)$-ary operation on $U$ by putting $\nmid u_{k}^{0}=\dagger t_{k}^{0}$, where $t_{i}$ is $\left\{x_{i}\right\}$ if $u_{i}$ is $x_{i}$, and $t_{i}$ is $u_{i}$ if not. The new operation is clearly isomorphic to the old, and is therefore $(k, k)$-associative. (1) becomes

$$
\dagger^{n} x_{n k}^{0}=* x_{n k}^{0} \text {. }
$$

Note. Although the main theorem is stated only in terms of associativity, and with the minimum reversibility and regularity requirements needed for the proof, much the same result would have been obtained if we had restricted our attention to regular reversible operations (i.e. operations which are $l$-regular and $l$-reversible for every $l$ ), for it is clear that if $*$ is regular and reversible, then so is $\dagger$. (Indeed, the importance of $(j, k)$-associativity is that it is the general form of associativity for a regular reversible operation.) This is the point of the equivalence $q$ : by identifying those elements of $T$ which necessarily behave alike under the operation, it preserves regularity.

If we now neglect regularity and reversibility, and simply require $\dagger$ to be $(k, k)$-associative, we can generalize the associativity of $*$ from $(1, k)$ to $(j, k)$, by replacing $q$ by the identity $i$. The restriction $j=1$ comes only in the application of Lemma $G$; but if $q=i$, the uniqueness of $\uparrow t_{k}^{0}$ is obvious, and Lemma $G$ is unnecessary. The theorem is, of course, no longer so closely analogous to the theorem of E. L. Post which inspired it. Stated in full, it is:

If $*$ is a $(j, k$-) associative $(\nu+1)$-ary operation on $S$, there is a set $U$ containing $S$ and $a(k, k)$-associative $(k+1)$-ary operation $\dagger$ on $U$ such that $\dagger^{\nu / k_{\mathbf{X}}}=*_{\mathbf{X}}$ for every $\mathbf{x}$ of $S^{\nu+1}$.

\section{Replacement theorems.}

I. Theorem. If (i) $*$ is $(j, k)$-associative, l-reversible, and $l^{\prime}$-reversible, (ii) $* a_{p}^{0} F_{\nu-r-1}^{1} c_{r-p}^{0}=* a G_{\nu-r-1}^{1} \mathrm{c}$, (iii) $p$, $q$, and $r$ are multiples of $j$ and either $p$ or $r-p$ is a multiple of $k$, and (iv) $l \leqq \nu-p-1$ and $l^{\prime} \geqq r$ $-p+1$, then for any $b_{q}^{0}$ and $d_{r-q}^{0}$ for which $r \geqq q \geqq 0$,

$$
* b F d=* b G d \text {. }
$$

Proof. $b_{q}^{0}$ is not null because $q \geqq 0$. * is $l$-reversible, where $l \leqq \nu$ $-p-1$. Therefore, for some $e_{\nu-p}^{1}$,

$$
b_{q}=* e a .
$$

Similarly, for some $h_{\nu-r+p}^{1}$,

$$
d_{0}=* c h \text {. }
$$


Therefore

$$
* b F d=* b_{q-1}^{0} * e a F * c h d_{r-q}^{1} .
$$

Now (4) is of the form " $q, 2 \nu+q-r$ ": that is, the second and third operation signs come after $q$ elements and after $2 \nu+q-r$ elements respectively in the formula. Now $q, 2 \nu+q-r, \nu-p+q$, and $\nu+q-r$ are all multiples of $j$. If $r-p$ is a multiple of $k$, so is $(2 \nu+q-r)$ $-(\nu-p+q)$. Then, by the theorem in $A$ note on continued products, J. London Math. Soc. vol. 27 (1952) pp. 239-241, (4) is equal to a continued product of the form " $q, \nu-p+q$ "; that is, to

$$
* b_{q-1}^{0} * e * a F \operatorname{ch} d_{r-q}^{1} \text {. }
$$

If, however, it is $p$ which is a multiple of $k$, then so are $q-(\nu-p+q)$ and $(2 \nu+q-r)-(\nu+q-r)$. (4) is now equal to a continued product of the form " $\nu+q-r, \nu-p+q$ "; that is, to

$$
* b_{q-1}^{0} e_{\nu-r}^{1} * e_{\nu-p}^{\nu-r+1} * a F \mathrm{ch} d_{r-q}^{1} .
$$

Now we may use (ii) to replace $* a F c$ by $* a G c$ in (5) or (6), which is the same thing as replacing $F$ by $G$. Reversing our argument, either of these is clearly equal to (4) with $F$ replaced by $G$, and this, by (2) and (3), is equal to $* b G d$.

J. A crucial point in the proof of Theorem I was the replacement of $b_{q}$ and $d_{0}$ by products in order to make the continued-product theorem applicable. If $F_{1}$ is a unit set, $F_{1}=\{f\}$ say, then (2) can be replaced by the statement: $* e_{\nu-p-1}^{1} a_{p}^{0} f=f$, provided that (iv) is modified to read $l \leqq \nu-p-2$. Then if $p+1$ (instead of $p$ ) is a multiple of $j$, and so on, the proof goes through as before. Writing $p$ instead of $p+1$, and $a_{p}^{1}$ and so on in place of $a_{p-1}^{0}$ and so on, we have the following result:

THEOREM. If (i), (iii), and (iv) are true, if $* a_{p}^{1} F_{p-r}^{1} c_{r-p}^{0}=* a G c$, if $F_{1}$ is a unit set, and if $r \geqq q$, then

$$
* b_{q}^{1} F d_{r-q}^{0}=* b G d \text {. }
$$

K. Similarly,

THEOREM. If (i), (iii), and (iv) are true, if $* a_{p}^{0} F_{\nu-r}^{1} c_{r-p}^{1}=* a G c$, if $F_{\nu-r}$ is a unit set, and if $q \geqq 0$, then

$$
* b_{q}^{0} F d_{r-q}^{1}=* b G d
$$

L. Combining $\mathrm{J}$ and $\mathrm{K}$, we have 
TheOREM. If (i), (iii), and (iv) are true, if $F_{1}$ and $F_{\nu \rightarrow r+1}$ are unit sets, and if $* a_{p}^{1} F_{\nu-r+1}^{1} c_{\tau-p}^{1}=* a G c$, then

$$
* b_{q}^{1} F d_{r-q}^{1}=* b G d \text {. }
$$

M. If, in J, $p=0$, the replacement of $f$ by *eaf is unnecessary. Then $F_{1}$ need not be a unit set. Moreover, if $*$ is $q$-regular we can prove a converse:

TheOREM. If $*$ is $(j, k)$-associative and $l$-reversible, where $l \geqq r+1$, and if $r$ is a multiple of $k$ and $q$ of $j$, then

$$
* F_{\nu \rightarrow r}^{1} c_{r}^{0}=* G c
$$

implies that

$$
* b_{q}^{1} F d_{r-q}^{0}=* b G d \text {. }
$$

If, in addition, * is q-regular, then (2) implies (1).

Proof. Let $d_{0}=* c h_{\nu}^{r+1}$. Using this, associativity, and (1),

$$
\begin{aligned}
* b F d & =* b F * c h d_{r-q}^{1} \\
& =* b * F c h d_{r-q}^{1} \\
& =* b * G c h d_{r-q}^{1} \\
& =* b G * c h d_{r-q}^{1} \\
& =* b G d .
\end{aligned}
$$

Conversely, if (2) is true and * is $q$-regular, then

$$
* b F * c h d_{r-q}^{1}=* b G * c h d_{r-q}^{1} \text {. }
$$

Therefore

$$
* b * F \operatorname{ch} d_{r-q}^{1}=* b * G c h d_{r-q}^{1}
$$

Therefore

$$
* F \mathrm{c}=* G \mathrm{c}, \quad \text { because } * \text { is } q \text {-regular. }
$$

N. Similarly,

ThEOREM. If $r$ is a multiple of $k$ and $q$ of $j$, and if $*$ is l-reversible where $l \leqq \nu-r-1$, then $* a_{r}^{0} F=* a G$ implies that $* b_{q}^{0} F d=* b G d$; and if $*$ is also $(\nu+q-r)$-reversible, the latter implies the former.

3. Identities.

O. Theorem. If a $(\nu+1)$-ary $(j, k)$-associative operation $*$ is either 0 - and $\nu$-regular or 0 - and m-regular or $(\nu-m)$ - and $\nu$-regular, or 
$(\nu-m)$ - and m-regular, where $m$ is a nonzero multiple of $k$, then $* e a=a$ implies that $* e x=x$ and $* a e=a$ implies that $* x e=x$.

Proof. By Theorem $\mathrm{B}, * e a=a$ implies that $* x e_{\nu}^{\nu-m+2} e_{\nu-m+1}^{1}=x$ which, by note $\mathrm{B}$, implies that $* e x=x$. Similarly for the second part.

Corollary. If $a(j, k)$-associative operation $*$ is 0 - and $\nu$-regular, then $* e a=a$ implies $* e x=x$, and $* a e=a$ implies $* x e=x$.

Proof. Put $m=\nu$ in the theorem.

P. Theorem. If $a(j, k)$-associative operation $*$ is 0 - and $\nu$-regular, and if $m$ is a multiple of $k$, then $* a e=a$ implies that $* x e_{\nu}^{m+1} e_{m}^{1}=x$, and $* e a=a$ implies that $* e_{\nu}^{\nu-m+1} e_{\nu-m}^{1} x=x$.

Proof. If $m=0$, this follows from corollary $\mathrm{O}$.

If $m \neq 0$, then $* e_{\nu}^{m} e_{m-1}^{1} x=x$, by note $\mathrm{B}$. Therefore

$$
* e_{\nu}^{m} e_{m}^{1}=e_{m}
$$

Therefore

$$
* x e_{\nu}^{m+1} e_{m}^{1}=x
$$

Similarly for the second part.

Q. Theorem. If a $(j, k)$-associative operation $*$ is 0 - and $\nu$-regular, and $* e_{m-1}^{0} x e_{\nu}^{m+1}=x$, then $* x e_{m-1}^{1} e_{0} e_{\nu}^{m+1}=* e_{m-1}^{0} e_{\nu} e_{\nu-1}^{m+1} x=x$. If, in addition, $m$ is a multiple of $k$, then $* e_{\nu} e_{\nu-1}^{m+1} x e_{m-1}^{1} e_{0}=x$.

Proof. $* e_{m-1}^{0} e_{0} e_{\nu}^{m+1}=e_{0}$. Therefore, by Corollary $\mathrm{O}$,

$$
\begin{gathered}
* x e_{m-1}^{1} e_{0} e_{\nu}^{m+1}=x, \text { and } \\
* e_{m-1}^{0} e_{\nu} e_{\nu}^{m+1}=e_{\nu} .
\end{gathered}
$$

Therefore, by Corollary $\mathrm{O}$,

$$
* e_{m-1}^{0} e_{\nu} e_{\nu-1}^{m+1} x=x .
$$

Putting $*_{\nu} e_{\nu-1}^{m+1} x e_{m-1}^{1} e_{0}$ in place of $y$ in the equation $y=* e_{m-1}^{0} y e_{\nu}^{m+1}$, we have

$$
\begin{aligned}
* e_{\nu} e_{\nu-1}^{m+1} x e_{m-1}^{1} e_{0} & =* e_{m-1}^{0} * e_{\nu} e_{\nu-1}^{m+1} x e_{m-1}^{1} e_{0} e_{\nu}^{m+1} \\
& =*^{2} e_{m-1}^{0} e_{\nu} e_{\nu-1}^{m+1} x e_{m-1}^{1} e_{0} e_{\nu}^{m+1} \\
& =* x e_{m-1}^{1} e_{0} e_{\nu}^{m+1} \\
& =x
\end{aligned}
$$


Corollary. If $k=1$ and $* e_{0} x e_{\nu}^{2}=x$, then $* a x e_{0} b=* a e_{0} x b$ for any a and $b$.

ProOF.

$$
\begin{aligned}
* a x e_{0} b & \left.=* a * e_{0} e_{\nu} e_{\nu-1}^{2} x e_{0} b \quad \text { (by (2) with } m=1\right) \\
& =* a e_{0} * e_{\nu} e_{\nu-1}^{2} x e_{0} b \\
& =* a e_{0} x b \quad(\text { by Theorem } Q \text { with } m=1)
\end{aligned}
$$

UNIVERSITY OF BRISTOL

\section{TWO THEOREMS ON FINITELY GENERATED GROUPS}

\section{EUGENE SCHENKMAN}

Let $G$ be a group generated by a finite subgroup $H$ and an element $b$ of finite order. If $H$ commutes elementwise with $b$ (for this we shall write $[h, b]=e$ for every $h \in H$ where $[h, b]$ designates $\left.h b h^{-1} b^{-1}\right)$, then clearly $G$ is finite and $b$ is in the center of $G$.

We consider here the case where, for every $h \in H,[[h, b] b]=e$, and prove the following theorem:

Theorem. Let $G$ be generated by the finite subgroup $H$ and the element $b$ of finite order and, for every $h \in H$, let $[[h, b] b]=e$. Then $G$ is finite and $b$ is in the nil radical of $G$.

Proof. For $i=1,2, \cdots, n$ let $h_{i}$ be the elements of $H$. Then $h_{i}^{-1} b h_{i}$ are all the conjugates of $b$; for $b h^{-1} b h b^{-1}=h^{-1} b h$ by virtue of the hypothesis $[[h, b] b]=e$.

It follows from the fact that a finite set of conjugates generate a finite normal subgroup (cf. [1]) that $b$ is contained in a finite normal subgroup $K$ of $G$. But $H$ is finite and hence so also is $G / K$; and then finally $G$ is finite.

Furthermore since $b$ is in the center of $K, b$ is in the nil radical of $G$ as was asserted.

We can deduce another result from the fact that $[[g, b] b]=e$ for every $g \in G$ implies that $b$ is in the center of a normal subgroup of $G$.

THEOREM. Let $G$ be a finitely generated group with the property that if $b_{1}, \cdots, b_{n}$ are the generators of $G$, then $\left[\left[g, b_{i}\right] b_{i}\right]=e$ for every $g \in G$ and for $i=1,2, \cdots, n$. Then $G$ is nilpotent of class at most $n$. If furthermore the $b_{i}$ are of finite order then $G$ is finite.

Received by the editors September 13, 1953. 